\title{
Conductivity Measurement of ZnO Nanowires Using the Powder-Solution-Composite Technique
}

\author{
S. Murali, ${ }^{a}$ S. Prasertpalichat, ${ }^{b}$ C. C. Huang, ${ }^{a}$ D. Cann, ${ }^{b}$ R. Yimnirun, ${ }^{c}$ \\ and J. F. Conley, Jr., ${ }^{\text {a, }, z}$ \\ ${ }^{a}$ School of Electrical Engineering and Computer Science, Oregon State University, Corvallis, Oregon 97331, USA \\ ${ }^{b}$ Materials Science, Department of Mechanical Engineering, Oregon State University, Corvallis, Oregon 97331, USA \\ ${ }^{c}$ School of Physics, Institute of Science, Suranaree University of Technology, Nakhon Ratchasima 30000, Thailand
}

\begin{abstract}
The powder solution composite (PSC) method was used to obtain the conductivity of $\mathrm{ZnO}$ nanowires from impedance spectroscopy measurements of $\mathrm{ZnO}$ nanowires dispersed in solution. The PSC method was calibrated by comparing impedance spectroscopy measurements of $\mathrm{ZnO}$ powder dispersed in solution with four-point probe measurements on bulk $\mathrm{ZnO}$ ceramic pellets. Conductivity values for $\mathrm{ZnO}$ nanowires obtained using this novel approach were found to be comparable to reported values obtained using two-point and four-point probe measurements of single or multiple nanowire devices isolated on a substrate. It is shown that application of the PSC method to $\mathrm{ZnO}$ nanowire solutions avoids the difficult process of forming electrical contacts to nanowires and allows for the non-destructive in-situ measurement of the average electrical properties of a large quantity of nanowires.

(C) 2011 The Electrochemical Society. [DOI: 10.1149/1.3615844] All rights reserved.
\end{abstract}

Manuscript submitted January 5, 2011; revised manuscript received June 28, 2011. Published July 28, 2011.

Determining the average conductivity of large numbers of quasi one dimensional structures such as nanowires (NWs) and carbon nanotubes remains a challenge. The most straightforward method of determining the conductivity of NWs is by running a series of I-V measurements on a NW device with two or more electrodes. However, application of electrical contacts to NWs is itself challenging. NW devices are still most often constructed via a "pick and place" type approach in which NWs in solution are randomly dispersed onto a substrate. This approach requires either sophisticated or low yield processes to fabricate electrodes for individual or multiple NW devices and is not suitable for rapid assessment of the average conductivity of a large number of NWs. If instead pre-fabricated electrodes are used, it has been shown that bending of NWs over the edge of the electrodes ${ }^{1}$ changes the strain on the NWs and affects the mobility and carrier concentration. ${ }^{2}$ The resistivity of free standing NWs was recently determined without forming electrodes using a low energy electron point source microscope; ${ }^{3}$ however, this technique may be difficult to implement in a production environment and may only be used for measurement of individual NWs.

A method of measuring conductivity that circumvents the tedious process of nanowire electrode formation and also allows simultaneous measurement of a large number of NWs is critically needed. AC-impedance spectroscopy is a non-destructive, precise, and highly repeatable technique that has been used to characterize ceramic powder solution composites. The powder-solution-composite (PSC) method was recently developed as a method for combining AC-impedance spectroscopy measurements with effective medium theory to determine the conductivity of a loose ceramic powder. ${ }^{4}$ The PSC method is based on measuring the AC-impedance spectra of "slurry composites" made from mixing the ceramic powder of unknown conductivity in a series of aqueous electrolytes spanning an appropriate range of known conductivity. Although the possibility for measuring ellipsoid particles and high aspect ratio fibers has been demonstrated, ${ }^{5,6}$ there have been no reports utilizing the PSC method to measure the conductivity of NWs.

In this work, we use the PSC method to assess the average electronic properties of $\mathrm{ZnO} \mathrm{NWs}$ in solution without fabrication or patterning of electrodes. The validity of the PSC method was first confirmed for powdered bulk $\mathrm{ZnO}$ ceramics via comparison to fourpoint probe measurements on $\mathrm{ZnO}$ sintered bars. The PSC method was then applied to determine the average conductivity of $\mathrm{ZnO}$ NWs suspended in $\mathrm{NaCl}$ solution. We demonstrate that the PSC technique can be used as a non-destructive technique for the charac-

\footnotetext{
* Electrochemical Society Active Member.

z E-mail: jconley@eecs.oregonstate.edu
}

terization of the average electrical properties of large numbers of NWs in solution.

Recently, AC dielectrophoresis, in which nanowires dispersed in solution are flowed across a substrate and aligned to lithographically defined electrodes with the aid of AC electric fields, ${ }^{7,8}$ has emerged as a promising method for NW integration. Before this method of NW integration can be scaled to production, techniques for rapidly assessing the quality of batches of nanowire solution will be required. This work shows that the PSC technique should be useful for in-situ assessment of the average quality of suspended NW solutions prior to their use on a device substrate.

\section{Experimental}

Sample preparation.-Bulk ceramic specimens were prepared from raw powders of $\mathrm{ZnO}$ (99.9\%, Alfa Aesar) that were ball milled for $6 \mathrm{~h}$ to reduce the particle size and dried overnight. The powder was then calcined at $950^{\circ} \mathrm{C}$ for $3 \mathrm{~h}$ and then uniaxially pressed under $100 \mathrm{MPa}$ into a $2.5 \mathrm{~cm}$-diameter pellet. The bulk $\mathrm{ZnO}$ pellet was subsequently sintered at $1100^{\circ} \mathrm{C}$ for $4 \mathrm{~h}$. X-ray diffraction was used to check the phase purity. The ceramic powder used in the resistivity measurements was obtained by pulverizing the sintered pellet by agate mortar and then filtrated with a $150 \mu \mathrm{m}$ diameter sieve.

A modified method for synthesis and harvesting $\mathrm{ZnO}$ NWs has been formulated in this research. As opposed to use of a catalyst ${ }^{9}$ or $\mathrm{ZnO}$ seed layer, ${ }^{10}$ a dense layer of $\mathrm{ZnO} \mathrm{NWs}$ was grown on a carbonized positive photoresist (Shipley S1818) nucleation layer ${ }^{11-14}$ using a conventional solid vapor transport method. First, a $2 \mu \mathrm{m}$ thick layer of photoresist was spin-coated onto a $1 \times 1$ (inch) (Ref. 2 ) $\mathrm{Si}$ substrate and then carbonized at $900^{\circ} \mathrm{C}$ for $60 \mathrm{~min}$ and 3 Torr under $150 \mathrm{sccm} \mathrm{N}_{2}$ flow. A 1:1 mixture of pure $\mathrm{ZnO}(99.9 \%$, Alfa Aesar) and graphite powders $(99.9995 \%$, Alpha Aeser) was then prepared and placed in a tube furnace at $925^{\circ} \mathrm{C}$. A $150 \mathrm{sccm}$ flow of $\mathrm{N}_{2}$ was used to carry the $\mathrm{Zn}$ vapor (produced by carbothermal reduction $^{9}$ ) downstream to the substrates at $770{ }^{\circ} \mathrm{C}$. Oxygen gas at $1 \mathrm{sccm}$ was introduced $5 \mathrm{~cm}$ from the samples to aid NW growth. After $3 \mathrm{~h}$ of growth, the substrate was quenched in air and a thick white layer of free-standing $\mathrm{ZnO} \mathrm{NWs}$ (nano-carpet) was peeled off from the carbonized photoresist (see Fig. 1). A large quantity $(\sim 50 \mathrm{mg})$ of NWs was grown. The average length and diameter of $10 \mu \mathrm{m}$ and $45 \mathrm{~nm}$, respectively, were determined by using a dual beam field emission scanning electron microscope (Quanta 3D SEM, FEI). Harvesting of the NWs into solution was performed via sonication of the nano-carpet in de-ionized (DI) water for $1 \mathrm{~min}$. Finally, as described in the next section, $\mathrm{NaCl}$ was dissolved into the NW/DI water suspension to form solutions over a wide range of conductivity. 

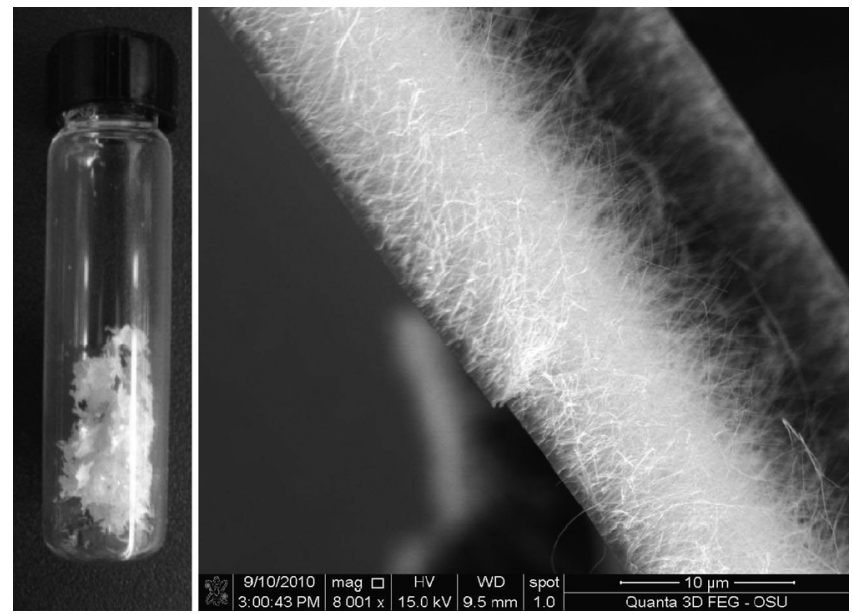

Figure 1. (Left) $50 \mathrm{mg}$ of $\mathrm{ZnO} \mathrm{NWs}$ grown via vapor solid transfer using carbonized photoresist as a nucleation layer, (right) peeled off sheet of free standing NW carpet.

Electrical measurements.-The bulk ceramic conductivity was obtained using a four-point DC conductivity measurement. ${ }^{15}$ The sintered pellet was cut into a rectangular bar $[10 \times 10 \times 5 \mathrm{~mm}$ (Ref. $3)$ ] with small notches made at approximately $1 / 3$ and $2 / 3$ positions along the length of the bar. The outer electrodes were made by applying silver paint at the both ends of the bar while the inner electrodes were made by wrapping two gold wires tightly and equally spaced around the marked notches. ${ }^{16}$ The four-point DC measurement was implemented by applying a current (Model 195A, Keithley) to the outer electrodes and measuring the voltage drop across the inner gold wires using a digital multi-meter (Fluke 179 multimeter, Optimum Energy Products Ltd.).

For the PSC measurements, a suspended $\mathrm{NaCl}$ solution of either pulverized $\mathrm{ZnO}$ powder or $\mathrm{ZnO}$ NWs was used. There are two primary concerns in preparing the suspensions. First, the solution must not react with the material under test. ${ }^{4,5}$ Second, in order to determine the conductivity of the material under test, it must be easy to adjust the conductivity of the solution over a range spanning the expected conductivity of the sample. $\mathrm{NaCl}$ has been shown to allow a wide range of solution conductivity, from $10^{-4}$ to $0.5 \mathrm{~S} \mathrm{~cm}^{-1}$. A series of $\mathrm{NaCl}$ solutions with the conductivity ranging from $1.4 \times 10^{-4} \mathrm{~S} \mathrm{~cm}^{-1}(0.001 \mathrm{M})$ to $2.8 \times 10^{-1} \mathrm{~S} \mathrm{~cm}^{-1}(0.3 \mathrm{M})$ were prepared by dissolving $\mathrm{NaCl}(99.5 \%$, Alfa Aesar) in DI water. Each of these solutions was then mixed with either pulverized $\mathrm{ZnO}$ powders of approximately $40 \%$ volume fraction or $\mathrm{ZnO}$ NWs of approximately $1 \%$ volume fraction.

Prepared solutions were placed into an apparatus consisting of a polyethylene tube $(4.7 \mathrm{~mm}$ inner diameter $\times 12.5 \mathrm{~mm}$ length $)$ and two stainless steel electrodes. ${ }^{4}$ The electrodes were polished with silicon carbide abrasive paper (1200/P2500, Buehler company) until the surface appeared shiny and smooth. Note that attention should be paid to the electrode surface roughness since it has been shown to have an effect on the impedance spectra, especially at low frequencies. ${ }^{17}$ The inner electrode spacing in this experiment was 10 $\mathrm{mm}$. A 1296 Hewlett Packard low-frequency impedance spectrometer in conjunction with a Solartron 1260 frequency analyzer was used for measuring the ac voltage response of the system. The frequency range used was from $10 \mathrm{~Hz}$ to $25 \mathrm{MHz}$. Simulations of impedance spectra were conducted using Zview Impedance Simulation software.

\section{Results and Discussion}

The PSC technique ${ }^{4-6,18}$ is based on impedance spectroscopy (IS) and effective medium theory. There are three basic requirements of the system to be analyzed: (1) a moderately conductive matrix (in our case an $\mathrm{NaCl}$ solution), (2) highly conducting particles dispersed in the solution (here either $\mathrm{ZnO}$ powder or $\mathrm{ZnO}$ NWs), and (3) a relatively high impedance layer between the solution and particle which can either be an oxide shell, Schottky barrier formation, or, as is likely in our case, an electrical double layer. AC impedance spectra are typically plotted in the Nyquist representation (negative imaginary impedance vs. real impedance) with increasing frequency from right to left (frequency markers, included as small numbers along each plot, represent the logarithm of the frequency).

Shown in Fig. 2 is a Nyquist plot of simulated and experimental impedance measurements of $0.001 \mathrm{M} \mathrm{NaCl}$ with $40 \%$ volume fraction $\mathrm{ZnO}$ ceramic powder. (Due to the difficulties associated with obtaining four-point conductivity measurements for large numbers of NWs, the validity of the PSC method for $\mathrm{ZnO}$ was first confirmed by comparing the conductivity obtained using the PSC method on $\mathrm{ZnO}$ powders with the conductivity obtained using four-point measurements of $\mathrm{ZnO}$ ceramics.) Each electrical component in the system (e.g. electrode, solution/composite) is represented by a semicircular arc in the plot. ${ }^{19}$ The presence of the high impedance layer between the particle and the solution is critical. The electrical double-layer creates a "frequency-switchable" interfacial impedance at the $\mathrm{ZnO}$ particle surface (described fully in Refs. 6 and 18) that results in the division of the solution impedance arc into two separate arcs on a Nyquist plot. As a result, two cusps are typically observed in impedance spectra for these types of systems. The cusp at low frequency excitation (denoted as $\mathrm{R}_{\mathrm{DC}}$ on the right side of Fig. 2) occurs because the high impedance layer on the particle surface is insulating at low frequencies, which makes the particles behave as if they are insulating with respect to the solution. At higher frequencies however, capacitive displacement currents can short out this high impedance layer and pass through to the particles, making the particles conductive with respect to the solution. Therefore, the cusp resistance (denoted as $\mathrm{R}_{\text {cusp }}$ on the left side of Fig. 2), represents the composite resistance without the effect of the high

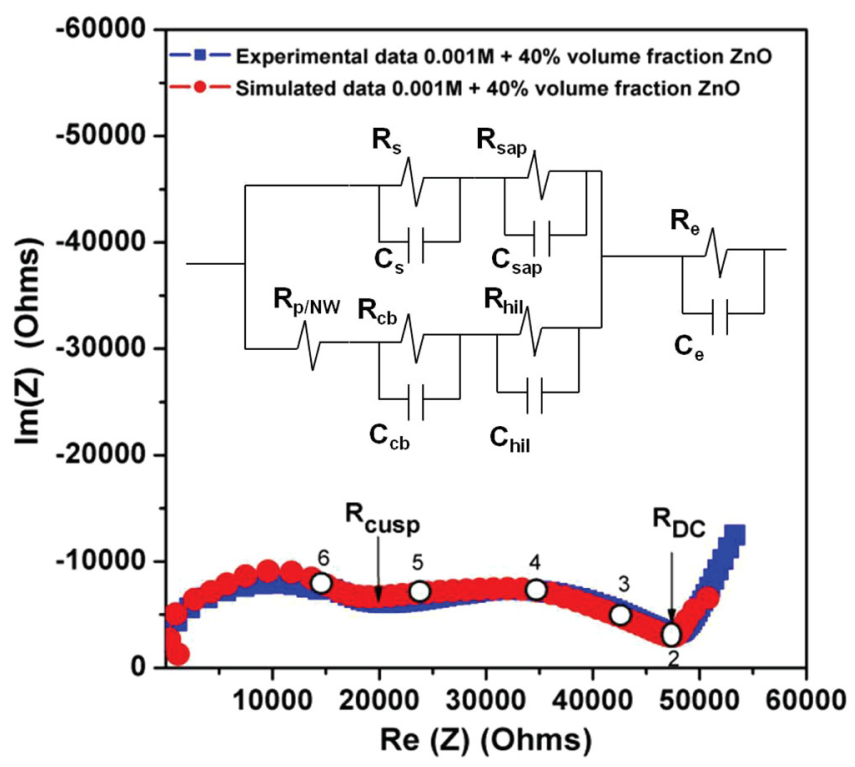

Figure 2. (Color online) Nyquist plot representation of the simulated (red circles) and experimental (blue squares) impedance spectra for a $0.001 \mathrm{M}$ $\mathrm{NaCl}$ solution with $40 \%$ volume fraction of $\mathrm{ZnO}$ ceramic powder. The small numbers along each plot are frequency markers that represent the $\log$ (frequency). Shown as an inset is the equivalent circuit model (adapted from and discussed in detail in Ref. 4) used in the work for simulating PSC impedance spectra, where $\mathrm{R}_{\mathrm{p} / \mathrm{NW}}$ represents the particle or NW resistance, $s$ represents the solution, sap represents solution around the particle or NWs, $c b$ represents current bunching, hil represents the high impedance layer surrounding the particles or NWs, and $e$ represents the electrode. 
impedance surface layer and is used for extracting the conductivity of particle. The conductivity calculated from the impedance spectra is thus a sum of both ionic as well as electronic conductivity. ${ }^{5}$

Shown as an inset in Fig. 2, is the equivalent circuit used to simulate the impedance spectra in this work. It is essentially the same as the model proposed by Ingram and Mason and the details are described fully in Ref. 4. In brief, at low measurements frequencies, the resistance of the high impedance layer, $\mathrm{R}_{\text {hil }}$, surrounding the particles is high and the bottom branch of the circuit is effectively open. At higher frequencies, the displacement currents short the high impedance layer and current flows through the bottom branch. In order to compensate for non-homogeneities in the real system, the capacitors in this model are replaced by constant phase elements, as explained in Ref. 4.

The PSC method has been demonstrated for measuring the conductivity of insulating and conducting particles of various shapes. ${ }^{4-6,18,20,21}$ A requirement for the PSC method is knowledge about the size and shape of the ceramic powder being analyzed, since this knowledge allows one to choose the appropriate effective medium theories for analyzing the conductivity of the system. The $\mathrm{ZnO}$ ceramic powder used in this study is roughly spherical and the model proposed by Bruggeman is suitable ${ }^{4}$

Spherical (ceramic powder): $\frac{\left(\sigma_{c} / \sigma_{s}\right)-\left(\sigma_{p} / \sigma_{s}\right)}{\left(\sigma_{c} / \sigma_{s}\right)^{1 / 3}\left(1-\left(\sigma_{p} / \sigma_{s}\right)\right)}=(1-f)$

The ZnO NWs, however, have an approximately ellipsoid shape with a broad range of sizes, and the model proposed by Fricke is used instead ${ }^{4,5}$

$$
\text { Ellipsoid (Wires): } \quad \sigma_{p}=\sigma_{c}-\frac{\left(\sigma_{s}-\sigma_{p}\right)}{1+\frac{\rho}{3} \sum_{\alpha} \frac{\left(\sigma_{s}-\sigma_{p}\right)}{\sigma_{p}-\eta_{\alpha} \cdot \sigma_{s}}}(1-f)
$$

In Eqs. 1 and 2, $\sigma_{c}, \sigma_{p}$, and $\sigma_{s}$ are the conductivity of the composite, particles or NWs, and solution, respectively; $f$ is the volume fraction of the suspended phase; $\eta_{\alpha}$ is the form factor which depends on the ellipsoid ratio; and $\rho$ is the density of $\mathrm{ZnO}$.

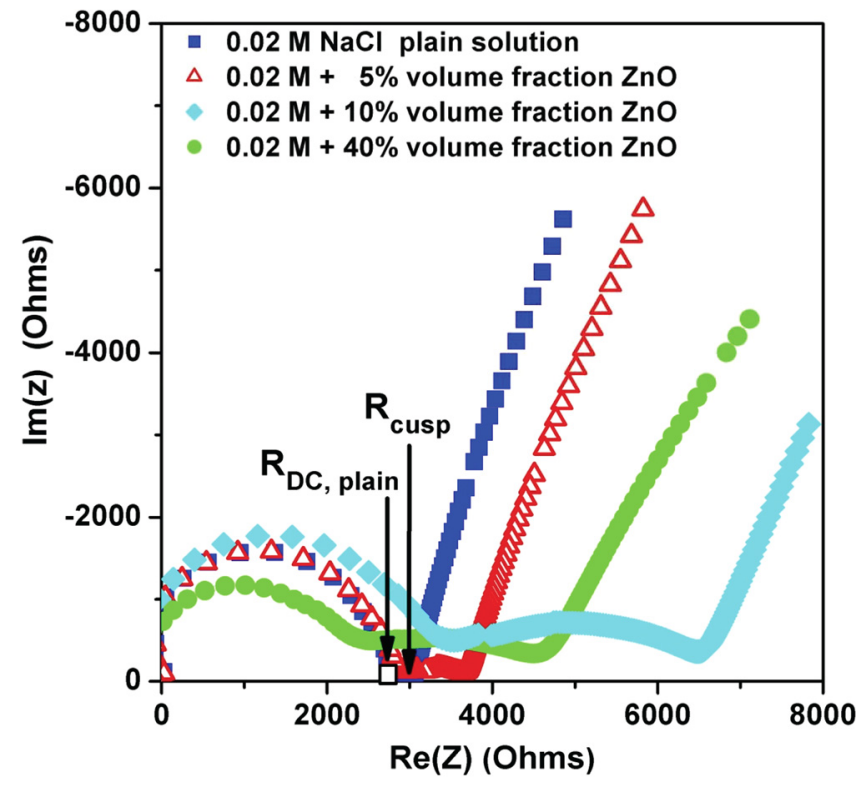

Figure 3. (Color online) Nyquist plot representation of the impedance spectra of a plain $0.02 \mathrm{M} \mathrm{NaCl}$ solution (blue squares), and $0.02 \mathrm{M} \mathrm{NaCl}$ composite solutions containing either 5\% (red triangles), $10 \%$ (cyan diamonds), or $40 \%$ (green circles) volume fraction of $\mathrm{ZnO}$ ceramic powder. The small numbers along each plot represent log (frequency).
Theoretically, the particle or NW conductivity $\left(\sigma_{p}\right)$ can be calculated directly from Eqs. 1 and 2 when $\sigma_{c}$ and $\sigma_{s}$ are measured. However, the single point datum can lead to a large error in determining $\sigma_{p}$ due to uncertainties in (i) controlling the volume fraction from the loss of some powder/water when the electrodes are squeezed before running each measurement and (ii) reading the resistance from the Nyquist plot for each individual measurement. ${ }^{4}$ These problems can be addressed by performing an experiment over a wide range of $\sigma_{s}$. This is the basis for PSC method. If a series of data can be collected throughout the regime where the solution and particle conductivity are comparable $\left(\sigma_{p}-\sigma_{s}\right)$, a crossover point where $\sigma_{s}=\sigma_{p}$ (obtained from a plot of $\log \left[\sigma_{s}\right]$ vs. $\log \left[\sigma_{c}\right]$ ) will be observed. ${ }^{4,5}$ This crossover point allows a more accurate determination of $\sigma_{p}$ since at this point, $\sigma_{c}$ is equivalent to both $\sigma_{p}$ and $\sigma_{s}$ $\left(\sigma_{c}-\sigma_{p}-\sigma_{s}\right)$ and the volume fraction term in Eqs. 1 and 2 will not affect the conductivity.

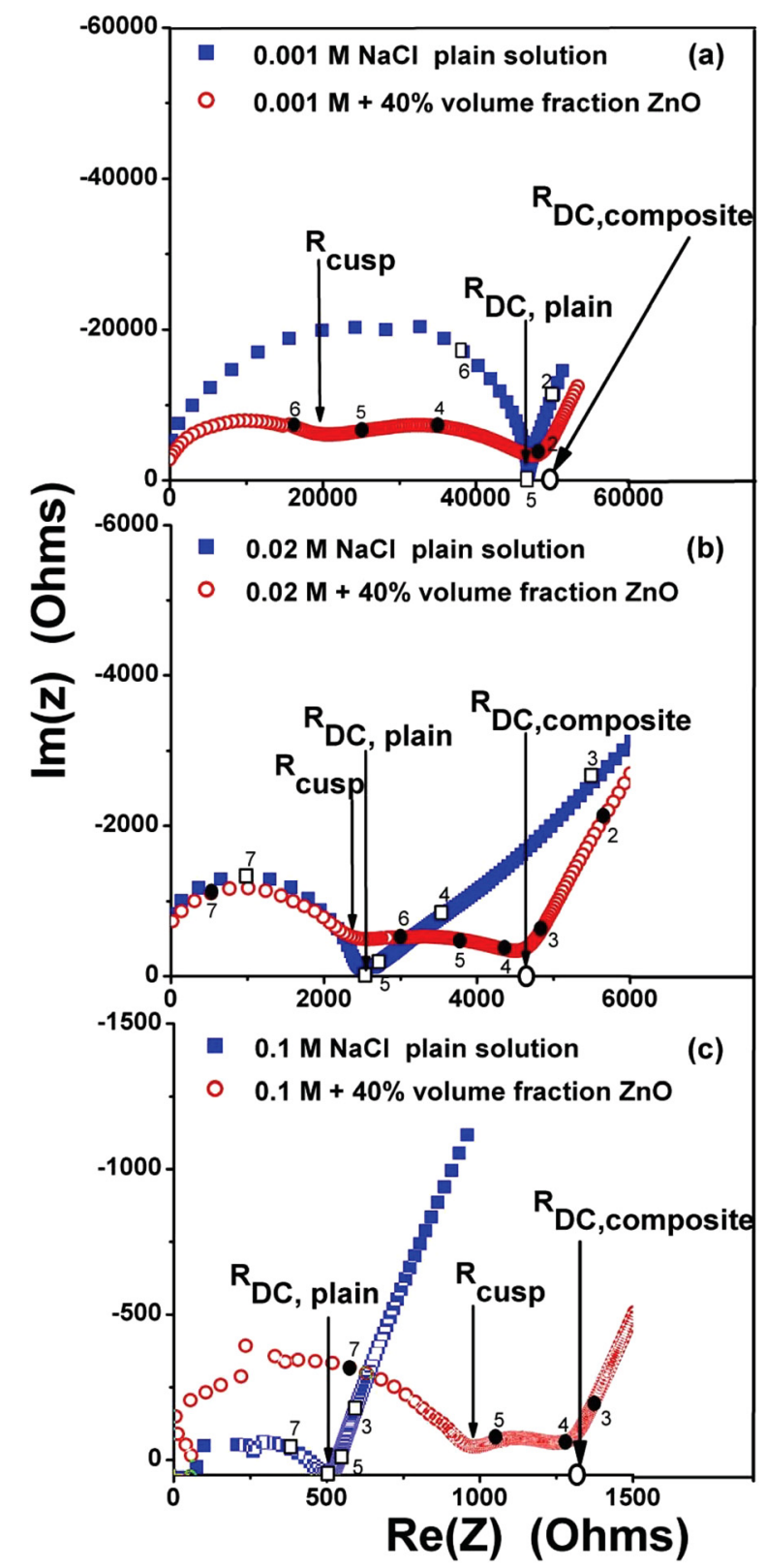

Figure 4. (Color online) Nyquist plot representation of the impedance spectra of $40 \%$ volume fraction $\mathrm{ZnO}$ powder/solution composites of (a) $0.001 \mathrm{M}$ $\mathrm{NaCl}$, (b) $0.02 \mathrm{M} \mathrm{NaCl}$, and (c) $0.1 \mathrm{M} \mathrm{NaCl}$. The small numbers along each plot represent $\log$ (frequency). 
A parallel percolation path may exist in the equivalent circuit ${ }^{4}$ in Fig. 2. As discussed in Ref. 22, as long as the conductivity of this particle-particle conduction path is significantly lower than the conductivity of the solution-particle path at the crossover point in a plot of $\log \left[\sigma_{s}\right]$ vs. $\log \left[\sigma_{c}\right]$, it will have a negligible effect on the PSC analysis. Agglomeration or settling of the particles may contribute to percolation. If the degree of agglomeration does not change dramatically from sample to sample, agglomeration should have a negligible effect on the crossover point. ${ }^{22}$ To minimize any potential impact of agglomeration and ensure uniform dispersal of the nanowires, all nanowire $/ \mathrm{NaCl}$ solutions were agitated for 2 min prior to each measurement. All impedance measurements (which last approximately $5 \mathrm{~min}$ ) were performed immediately after this procedure. As a control, a set of measurements that was performed under constant agitation was found give the same results as the measurements performed with agitation prior to the measurement.

Shown in Fig. 3 are impedance spectra of $0.02 \mathrm{M} \mathrm{NaCl}$ solutions either plain (without $\mathrm{ZnO}$ powder) or mixed with a $5 \%, 10 \%$, or $40 \%$ volume fraction of $\mathrm{ZnO}$ powder. (The $0.02 \mathrm{M} \mathrm{NaCl}$ concentration was chosen due to its matching conductivity with the bulk $\mathrm{ZnO}$ conductivity obtained from four-point probe measurements of sintered ceramic bars.) It is seen that $R_{\text {cusp }}$ matches well the solution resistance $\left(\mathrm{R}_{\mathrm{DC}}\right.$, plain $)$ with only a small deviation $(<15 \%$ error $)$, regardless of the volume fraction of $\mathrm{ZnO}$ ceramic powder. The error may be due to the length measurement of the solution in polyethylene tube or small air bubbles within the solution. These results indicate that, at the crossover point, the composite resistance has little dependence on volume fraction, as postulated but not shown in Ref. 4.

Shown in Figs. $4 \mathrm{a}-4 \mathrm{c}$ are AC impedance spectra for both plain $\mathrm{NaCl}$ solutions and powder composite solutions $(\mathrm{NaCl}$ solution $+40 \%$ volume fraction of $\mathrm{ZnO}$ ceramic powder) of 0.001 , 0.02 , and $0.1 \mathrm{M}$, respectively. The impedance spectra of the plain $\mathrm{NaCl}$ solution exhibited two arcs for all $\mathrm{NaCl}$ concentrations, confirming the: the left arc represents the bulk component, while the right arc corresponds to the electrode. $\mathrm{R}_{\mathrm{DC}}$, plain is indicated by the $\mathrm{x}$-axis intersection between the electrode and bulk arcs and also corresponds to the DC measurement. ${ }^{4}$

In Fig. $4 a$, it is seen that $R_{\text {cusp }}$ is much lower than $R_{D C \text {,plain, indi- }}$ cating that the particle is much more conductive than the solution $\left(\sigma_{p}>\sigma_{s}\right)$. As $\mathrm{NaCl}$ concentration increases, both $\mathrm{R}_{\mathrm{DC} \text {,plain }}$ and $\mathrm{R}_{\text {cusp }}$ decrease. Figure $4 \mathrm{~b}$ shows the nearly perfect coincidence between

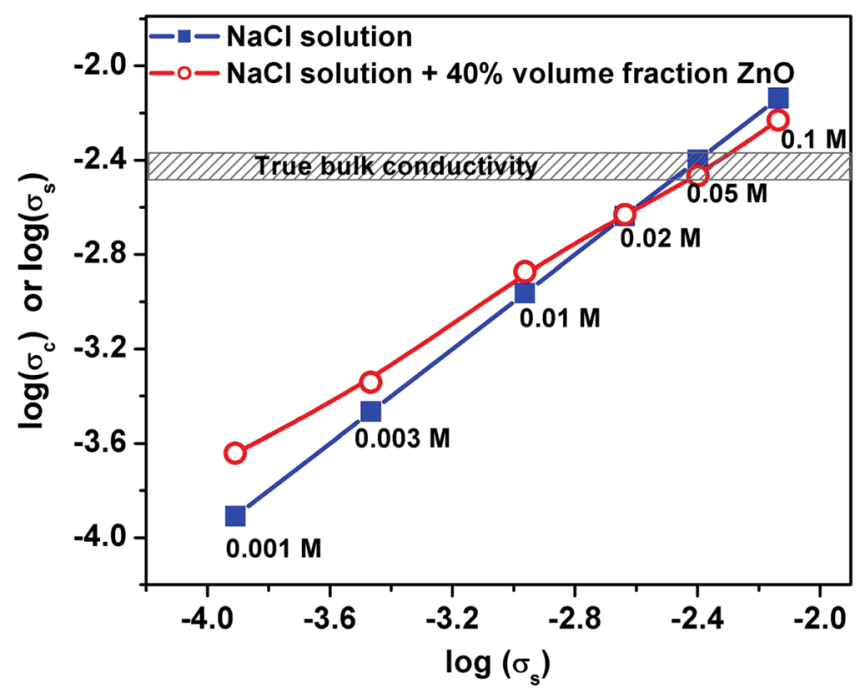

Figure 5. (Color online) Plot of $\log \left(\sigma_{c}\right)$ and $\log \left(\sigma_{s}\right)$ vs. $\log \left(\sigma_{s}\right)$ for a $40 \%$ volume fraction $\mathrm{ZnO}$ ceramic powder solution (open circles) and a plain $\mathrm{NaCl}$ solution (squares), respectively. The crossover point indicates the conductivity of the $\mathrm{ZnO}$ powder. The horizontal gray bar, labeled true bulk conductivity, is the conductivity range obtained from four-point probe measurements on ceramic bar samples.

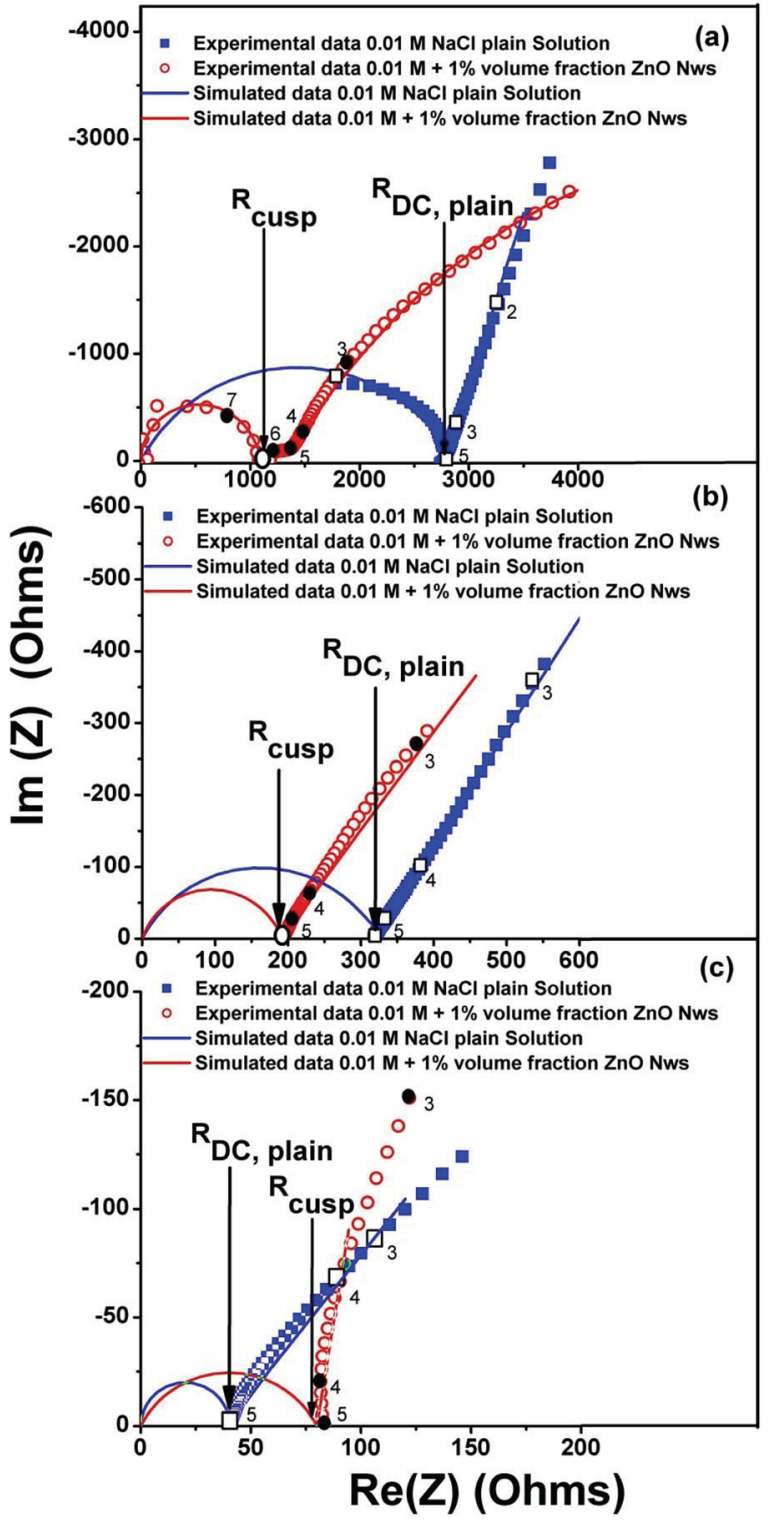

Figure 6. (Color online) Nyquist plot representation of the impedance spectra of plain and $1 \% \mathrm{ZnO}$ nanowire/solution composites of (a) $0.01 \mathrm{M}$, (b) 0.1 $\mathrm{M}$, and (c) $1 \mathrm{M} \mathrm{NaCl}$. The solid lines are simulated using the equivalent circuit shown in Fig. 2. The small numbers along each plot represent log (frequency).

$\mathrm{R}_{\mathrm{DC} \text {,plain }}$ and $\mathrm{R}_{\text {cusp }}$ for the $0.02 \mathrm{M} \mathrm{NaCl}$ solution and composite solution $\left(\sigma_{p}-\sigma_{s}\right)$, respectively. However, if $\mathrm{NaCl}$ concentration increases beyond this point, the solution becomes more conductive than the particle $\left(\sigma_{s}>\sigma_{p}\right)$. This situation is seen in Fig. 4c and is indicated by $\mathrm{R}_{\mathrm{DC} \text {,plain }}<\mathrm{R}_{\text {cusp. }}$. In summary, Fig. 4 demonstrates that the set of $\mathrm{NaCl}$ solution concentrations covers the regime in which the particle and solution conductivity are comparable.

Shown in Fig. 5 is a plot of $\log \left(\sigma_{c}\right)$ vs. $\log \left(\sigma_{s}\right)$ for each individual experiment from the data in Fig. 4. The crossover point in Fig. 5 yields $\sigma_{p}=2.6 \times 10^{-3} \pm 5 \times 10^{-4} \mathrm{~S} \mathrm{~cm}^{-1}$, which is close to the average sintered bar value of $3.69 \times 10^{-3} \mathrm{~S} \mathrm{~cm}^{-1}$ determined from our four-point measurements (shown as a gray horizontal bar in Fig. 5). The close match between the conductivity obtained from impedance measurements of $\mathrm{ZnO}$ powder and four point probe measurements of a sintered $\mathrm{ZnO}$ ceramic bar, suggest that the PSC method can also be a reliable method for measuring the conductivity of NWs.

Shown in Figs. 6a-6c are AC impedance spectra for both plain $\mathrm{NaCl}$ solutions and composite $\mathrm{ZnO} \mathrm{NW}$ solutions $(\mathrm{NaCl}$ 


\begin{tabular}{|c|c|c|c|c|}
\hline Group & Conductivity $\left(\mathrm{S} \mathrm{cm}^{-1}\right)$ & Synthesis method (Precursor) & Selective Growth Agent & Dimension $(l \times d)$ \\
\hline This work & $1.2 \times 10^{-2}$ & Solid vapor $(\mathrm{ZnO}+\mathrm{C})$ & Carbonized photoresist & $l: 10 \mu \mathrm{m}, d: 45-50 \mathrm{~nm}$ \\
\hline $\operatorname{Liu}^{\mathrm{a}}($ Ref. 24) & 2 & Solid vapor $(\mathrm{ZnO})$ & - & $d: 60-100 \mathrm{~nm}$ \\
\hline $\mathrm{Umar}^{\mathrm{a}}$ (Ref. 25) & $4.1 \times 10^{-5}$ & Solid vapor (Zn) & - & $l: 10-30 \mu \mathrm{m}, d: 60-100 \mathrm{~nm}$ \\
\hline Suh $^{\mathrm{a}}$ (Ref. 26) & $4.3-11.5$ & Solid vapor $(\mathrm{ZnO}+\mathrm{C})$ & Zinc Acetate Dihydrate & $l: 5-6 \mu \mathrm{m}, d: 30-70 \mathrm{~nm}$ \\
\hline P-C Chang (Ref. 27) & $(8.8-119) \times 10^{-2}$ & Solid vapor $(\mathrm{ZnO}+\mathrm{C})$ & Sn Film & $l: 1-3 \mu \mathrm{m}, d: 18-190 \mathrm{~nm}$ \\
\hline Weber (Ref. 3) & $1.2 \times 10^{-2}$ & Solid vapor (Zn) & $6 \mathrm{H}-, 4 \mathrm{H}-\mathrm{SiC}$ & $d: 150 \mathrm{~nm}$ \\
\hline Sakurai $^{a}$ (Ref. 28) & 2.2 & Solid vapor $(\mathrm{ZnO}+\mathrm{C})$ & $\mathrm{Au}$ & $l: 1-50 \mu \mathrm{m}, d: 50-400 \mathrm{~nm}$ \\
\hline Scrymgeour (Ref. 29) & 100 & Solution based $\left(\mathrm{Zn}\left(\mathrm{NO}_{3}\right)_{2}\right)$ & $\mathrm{ZnO}$ & $l: 4 \mu \mathrm{m}$ \\
\hline
\end{tabular}

${ }^{a}$ Conductivity values calculated from reported mobility and carrier concentration.

solution $+1 \%$ volume fraction of $\mathrm{ZnO} \mathrm{NWs}$ ) for $\mathrm{NaCl}$ concentrations of $0.01,0.1$, and $1 \mathrm{M}$, respectively. The solid lines are simulated using the equivalent circuit shown in Fig. 2. A similar trend in impedance spectra is observed for NWs as was seen for the $\mathrm{ZnO}$ powder, in which $\sigma_{c}>\sigma_{s}$ at low $\mathrm{NaCl}$ solution concentration and $\sigma_{c}<\sigma_{s}$ at high $\mathrm{NaCl}$ solution concentration. For the $\mathrm{ZnO} \mathrm{NWs}$, however, the difference between $\mathrm{R}_{\mathrm{DC}}$ and $\mathrm{R}_{\text {cusp }}$ is not as clear due to the lower concentration of $\mathrm{NWs}$ in $\mathrm{NaCl}$ solution. This phenomenon was also observed in the ceramic powder solution in Fig. 3 and in other systems such as carbon nanotube solutions. ${ }^{23}$ As the concentration of powder (or NWs) is decreased, the high impedance layer between the matrix $(\mathrm{NaCl}$ in this case) and the $\mathrm{ZnO}$ powder $(\mathrm{NWs})$ will become insignificant (i.e. $\mathrm{NaCl}$ dominates the impedance response). When the $\mathrm{NaCl}$ concentration is low, as shown in Fig. 6a, the plain $\mathrm{NaCl}$ solution has a higher resistance than the $\mathrm{ZnO}$ NWs (judged from the intersection between $\mathrm{R}_{\text {cusp }}$ and $\mathrm{x}$-axis). When the $\mathrm{NaCl}$ concentration is increased, the resistance of the plain $\mathrm{NaCl}$ solution becomes lower than the $\mathrm{ZnO} \mathrm{NWs}$, as revealed in Fig. 6c. The close fit of the simulation (solid line) to the data shows that the equivalent circuit in Fig. 2 is still a valid description of the system.

As seen in the plot of $\log \left(\sigma_{\mathrm{c}}\right)$ vs. $\log \left(\sigma_{\mathrm{s}}\right)$ shown in Fig. 7, the crossover point for NW solutions occurs between 0.1 and $0.3 \mathrm{M}$. In order to observe the impedance difference between the pure and composite solutions clearly, the volume fraction should not be too low. Observation of the crossover point is an indication that the $1 \%$ $\mathrm{ZnO} \mathrm{NW}$ volume fraction is sufficient for the PSC method. From the crossover point, the conductivity of the NWs is estimated to be approximately $1.2 \times 10^{-2} \mathrm{~S} \mathrm{~cm}^{-1}$. The conductivity of NWs is

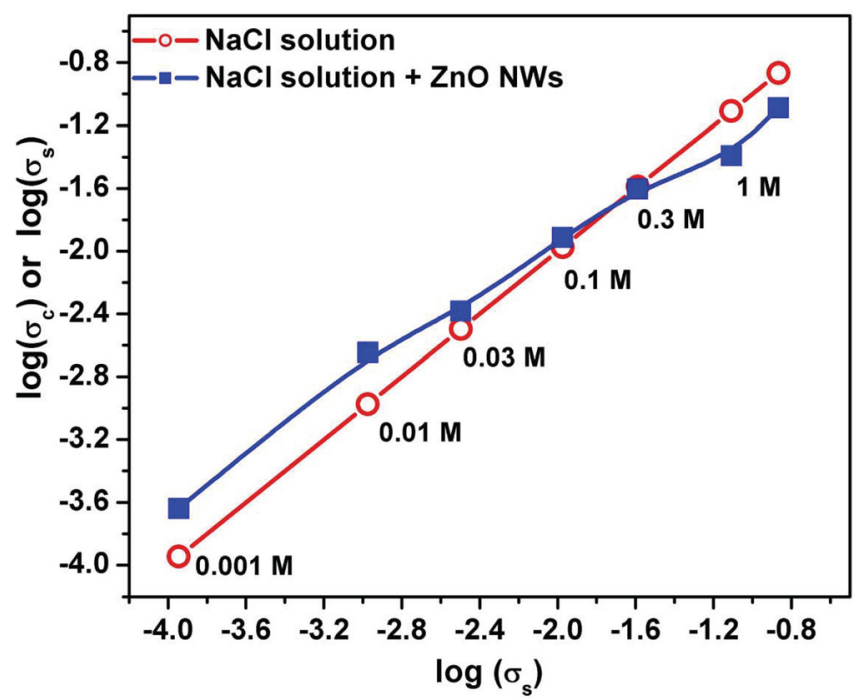

Figure 7. (Color online) Plot of $\log \left(\sigma_{\mathrm{s}}\right)$ and $\log \left(\sigma_{\mathrm{c}}\right)$ vs. $\log \left(\sigma_{\mathrm{s}}\right)$ for plain (squares) and $\mathrm{ZnO} \mathrm{NW}$ (open circles) $\mathrm{NaCl}$ solutions, respectively. The crossover point indicates the conductivity of the $\mathrm{ZnO}$ NWs. strongly dependent upon the growth process. As shown in Table I, the value of conductivity obtained from the PSC method is within the wide range of values reported for $\mathrm{ZnO} \mathrm{NW}$ devices.

\section{Conclusion}

Currently, a major challenge facing the use of NWs is in the wafer scale electrical integration of these quasi-one dimensional structures. A very promising integration technique that has recently emerged is AC dielectrophoresis. In dielectrophoresis, NWs suspended in solution are flowed over the surface of the device wafer and AC electric fields are used to align the NWs between lithographically defined electrodes. ${ }^{8}$ Before dielectrophoresis can be fully realized in a production environment, a non-destructive method of rapidly assessing the quality of batches of NW solution, prior to use on a device substrate, will be needed. The powder-solution-composite (PSC) technique was introduced by Ingram et al. ${ }^{4}$ as a method of using AC impedance spectroscopy to determine the conductivity of ceramic powders in solution. In this work, we have adapted the PSC method to characterize the conductivity of $\mathrm{ZnO}$ NWs in $\mathrm{NaCl}$ solution. We find that the PSC method is not dependent on particle volume fraction and allows in-situ measurements of NW solutions. We demonstrate that the PSC technique offers a nondestructive measurement platform for measuring the average conductivity of large quantities of NWs without forming electrical contacts to the NWs.

\section{References}

1. Y. W. Heo, D. P. Norton, L. C. Tien, Y. Kwon, B. S. Kang, F. Ren, S. J. Pearton, and J. R. LaRoche, Mater. Sci. Eng. Rep., 47, 1 (2004).

2. S.-S. Kwon, W.-K. Hong, G. Jo, J. Maeng, T.-W. Kim, S. Song, and T. Lee, Adv. Mater., 20, 4557 (2008).

3. D. H. Weber, A. Beyer, B. Völkel, A. Gölzhäuser, E. Schlenker, A. Bakin, and A. Waag, Appl. Phys. Lett., 91, 253126 (2007).

4. B. J. Ingram and T. O. Mason, J. Electrochem. Soc., 150, E396 (2003).

5. M. I. Bertoni, N. J. Kidner, T. O. Mason, T. A. Albrecht, E. M. Sorensen, and K. R. Poeppelmeier, J. Electroceram., 18,189 (2007).

6. J. M. Torrents, T. O. Mason, A. Peled, S. P. Shah, and E. J. Garboczi, J. Mater. Sci. 36, 4003 (2001).

7. P. A. Smith, C. D. Nordquist, T. N. Jackson, T. S. Mayer, B. R. Martin, J. Mbindyo, and T. E. Mallouk, Appl. Phys. Lett., 77, 1399 (2000).

8. E. M. Freer, O. Grachev, X. Duan, S. Martin, and D. P. Stumbo, Nat. Nanotechnol., 5, 525 (2010).

9. M. H. Huang, Y. Wu, H. Feick, N. Tran, E. Weber, and P. Yang, Adv. Mater., 13, $113(2001)$.

10. J. F. Conley, Jr., L. Stecker, and Y. Ono, Nanotechnology, 16, 292 (2005).

11. C. Cheng, M. Lei, L. Feng, T. L. Wong, K. M. Ho, K. K. Fung, M. M. T. Loy, D. Yu, and N. Wang, ACS Nano., 3, 53 (2009).

12. P. G. Li, W. H. Tang, and X. Wang, J. Alloys Compd., 479, 634 (2009).

13. C. C. Huang, B. D. Pelatt and J. F. Conley, Jr., Nanotechnology, 21, 195307 (2010).

14. B. Pelatt, C. C. Huang, and J. F. Conley, Jr., Solid-State Electron., 54, 1143 (2010).

15. B.-S. Hong, S. J. Ford, and T. O. Mason, Key Eng. Mater., 125-126, 163 (1997).

16. R. N. Blumenthal and M. A. Seitz, Electrical Conductivity in Ceramics and Glass, Part A, N. M. Tallan, Editor, p. 36, Marcel Dekker, New York (1974).

17. W. Cheng, G. Jin, and Y. Zhang, Russ. J. Electrochem., 9, 940 (2005).

18. L. Y. Woo, S. Wansom, A. D. Hixson, M. A. Campo, and T. O. Mason, J. Mater. Sci., 38, 2265 (2003).

19. J. R. Macdonald, Electrochim. Acta, 35, 1483 (1990). 
20. W. C. Sheets, E. S. Stampler, H. Kabbour, M. I. Bertoni, L. Cario, T. O. Mason, T. J. Marks, and K. R. Poeppelmeier, Inorg. Chem., 46, 10741 (2007).

21. G. H. Chan, B. Deng, M. Bertoni, J. R. Ireland, M. C. Hersam, T. O. Mason, R. P. Van Duyne, and J. A. Ibers, Inorg. Chem., 45, 8264 (2006).

22. K. H. Seo, J. H. Lee, J. J. Kim, M. I. Bertoni, B. J. Ingram, and T. O. Mason, J. Am. Ceram. Soc., 89, 3431 (2006).

23. S. Wansom, N. J. Kidner, L. Y. Woo, and T. O. Mason, Cem. Concr. Compos., 28, 509 (2006)

24. Y. Liu, Z. Zhang, H. Xu, L. Zhang, Z. Wang, W. Li, L. Ding, Y. Hu, M. Gao, Q. Li, et al, J. Phys. Chem. C, 113, 16796 (2009).
25. A. Umar, Y. K. Park, and Y. B. Hahn, J. Nanosci. Nanotech., 9, 2692 (2009).

26. D. I. Suh, S. Y. Lee, J. H. Hyung, T. R. Kim, and S. K. Lee, J. Phys. Chem. C, 112, 1276 (2008).

27. P.-C. Chang, C.-J. Chien, D. Stichtenoth, C. Ronning, and J. G. Lu, Appl. Phys. Lett., 90, 113101 (2007).

28. M. Sakurai, Y. G.Wang, T. Uemura, and M. Aono, Nanotechnology, 20, 155203 (2009).

29. D. A. Scrymgeour, C. Highstrete, Y.-J. Lee, J. W.-P. Hsu, and M. Lee, J. Appl. Phys., 107, 064312 (2010). 